\title{
Properties of the Molecular Gas in Starburst Galaxies and AGN
}

\author{
S. Mühle* \\ Joint Institute for VLBI in Europe \\ Postbus 2, 7990 AA Dwingeloo, The Netherlands \\ E-mail: muehle@jive.nl

\section{E.R. Seaquist} \\ Department of Astronomy and Astrophysics, University of Toronto \\ 50 St. George Street, Toronto, ON M5S 3H4, Canada \\ E-mail: seaquisteastro.utoronto.ca

\section{Henkel} \\ Max-Planck-Institut für Radioastronomie \\ Auf dem Hügel 69, 53121 Bonn, Germany \\ Email: p220hen@mpifr-bonn.mpg.de
}

\begin{abstract}
There is growing evidence that the properties of the molecular gas in the nuclei of starburst galaxies and in AGN may be very different from those seen in Galactic star forming regions and that a high kinetic temperature in the molecular gas may lead to a non-standard initial mass function in the next generation of stars. Unfortunately, among the fundamental parameters derived from molecular line observations, the kinetic temperature of the molecular gas in external galaxies is often not well determined due to a lack of suitable tracer molecules. We discuss the diagnostic power of selected transition lines of formaldehyde $\left(\mathrm{H}_{2} \mathrm{CO}\right)$, which can be used as a molecular thermometer as well as an excellent tracer of the molecular gas density. As a proof of concept, we present the results of our multi-transition line study of the $\mathrm{H}_{2} \mathrm{CO}$ emission from the prototypical starburst galaxy M82. Using our large velocity gradient model, we tightly constrain the physical properties of the dense gas in the prominent molecular lobes, completely independent of the standard "cloud thermometer" ammonia $\left(\mathrm{NH}_{3}\right)$ or other molecular tracers. Our results agree well with the properties of the high-excitation molecular gas component found in the most comprehensive CO studies. Our observations also indicate that there may be an asymmetry between the two molecular lobes.
\end{abstract}

The 9th European VLBI Network Symposium on The role of VLBI in the Golden Age for Radio Astronomy and EVN Users Meeting

September 23-26, 2008

Bologna, Italy

\footnotetext{
${ }^{*}$ Speaker.
} 

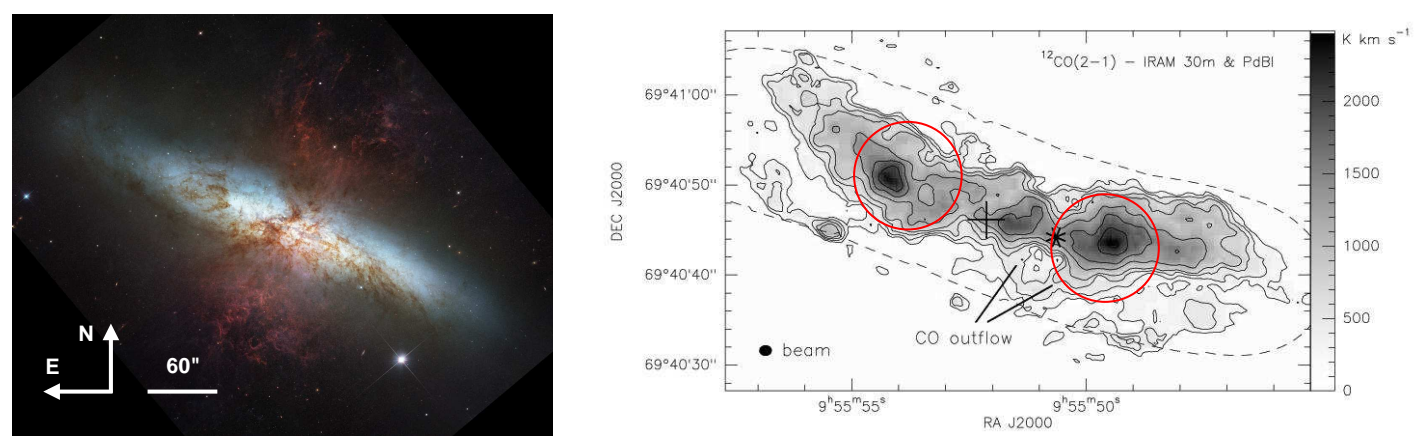

Figure 1: Left: Hubble Heritage image of the starburst galaxy M82 [14]. Right: High-resolution $\mathrm{CO}(2 \rightarrow 1)$ map of the circumnuclear molecular ring in M 82 [20]. The observed pointing positions of our study and the corresponding beam widths at $218 \mathrm{GHz}\left(11^{\prime \prime}\right)$ are marked by the solid circles.

\section{Introduction}

Star formation, one of the fundamental processes that shape the evolution of a galaxy and its surroundings, is thought to be fuelled by molecular gas, in particular by its dense component. Several observational studies have already shown the correlation between the dense molecular gas and star formation [e.g. 4]. But does star formation proceed in the same manner in all types of galaxies or does it depend significantly on the physical properties of the molecular gas? In recent years, more and more evidence has been found for the presence of a significant, or even dominant, warm molecular gas component in starburst galaxies and AGN [10,17] and for nonstandard initial mass functions in active environments [16,6]. Unfortunately, the physical properties of the molecular gas in external galaxies, in particular the kinetic temperature, are rarely well constrained.

The easily thermalized and optically thick $\mathrm{CO}(1 \rightarrow 0)$ and $\mathrm{CO}(2 \rightarrow 1)$ transitions would be a good temperature tracer, if the filling factor of extragalactic clouds was better known. The inversion lines of the symmetric top molecule ammonia $\left(\mathrm{NH}_{3}\right)$ are frequently used as "cloud thermometer" in our Galaxy. However, variations in the fractional abundance of up to three orders of magnitude within the disk of the Milky Way may indicate that ammonia traces only a specific component of the molecular gas. Other commonly observed molecules like HCN are good density tracers, but require an a priori knowledge of the kinetic temperature. Therefore, the dust temperature is often assumed to be a good measure of the kinetic temperature of the gas.

In this contribution, we present the results of our recent study on the physical properties of the molecular gas in the prototypical nearby starburst galaxy M 82, demonstrating the diagnostic power of selected para-formaldehyde lines for external galaxies [11]. Formaldehyde $\left(\mathrm{H}_{2} \mathrm{CO}\right)$ is a slightly asymmetric top molecule with a wealth of millimeter and submillimeter transitions in its subspecies para- $\mathrm{H}_{2} \mathrm{CO}$ and ortho- $\mathrm{H}_{2} \mathrm{CO}$ and shows only little variation in its fractional abundance in a variety of galactic environments [e.g. 5]. In general, line intensity ratios involving different $K_{a}$ ladders of energy levels are good tracers of the kinetic temperature, because the relative populations of these ladders are governed by collisions. By contrast, line ratios within a single $K_{a}$ ladder sensitively probe the gas density, once the kinetic temperature is known [7]. Thus, $\mathrm{H}_{2} \mathrm{CO}$ is not only one of the few direct molecular thermometers, but also an excellent tracer of the gas density. 

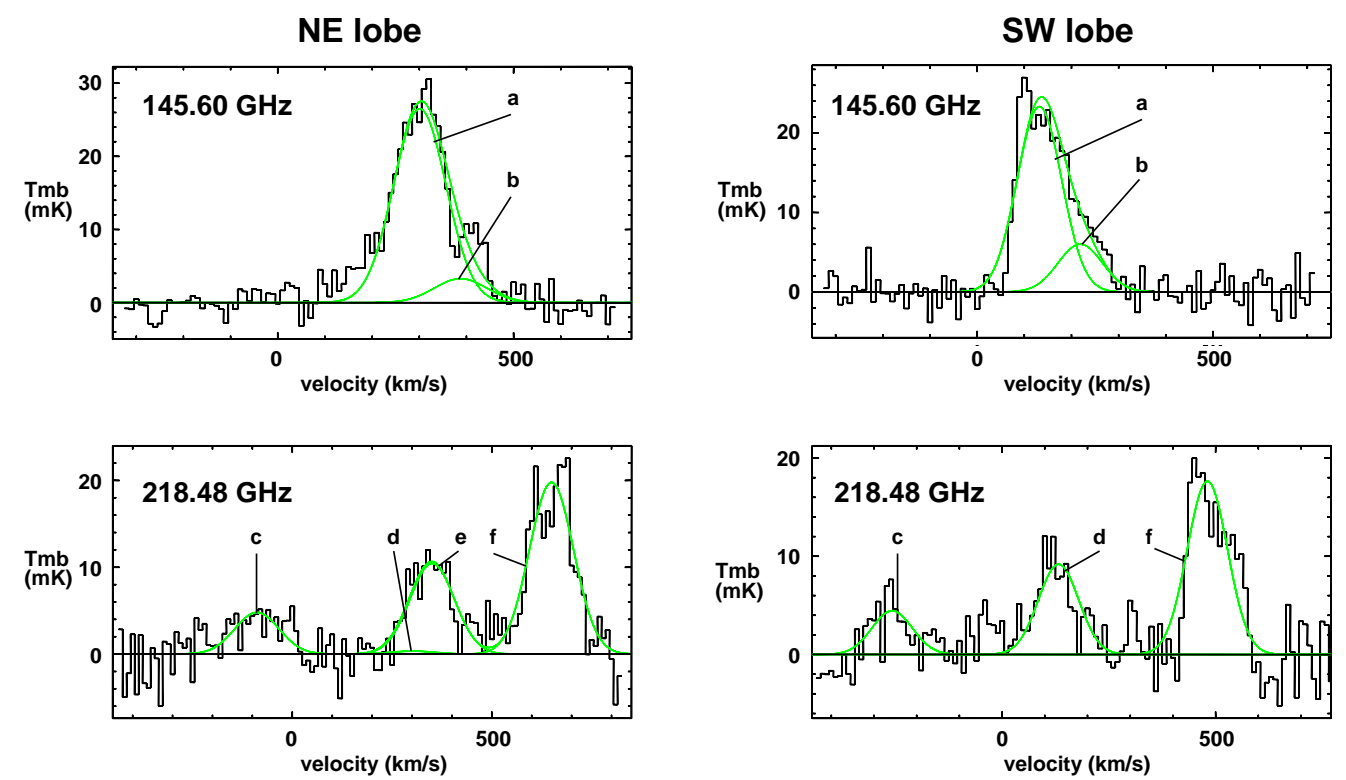

Figure 2: Observed spectra of the NE (left) and the SW lobe (right) of M 82. Each spectrum is labeled with the frequency the receiver was tuned to. Thus, the velocity scale of the $218.48 \mathrm{GHz}$ spectra refers to the $\mathrm{H}_{2} \mathrm{CO}\left(3_{22} \rightarrow 2_{21}\right)$ line. The $\mathrm{H}_{2} \mathrm{CO}\left(3_{03} \rightarrow 2_{02}\right)$ and the $\mathrm{H}_{2} \mathrm{CO}\left(3_{21} \rightarrow 2_{20}\right)$ transitions are offset by $348.5 \mathrm{~km} \mathrm{~s}^{-1}$ and $-389.7 \mathrm{~km} \mathrm{~s}^{-1}$, respectively, while the $\mathrm{CH}_{3} \mathrm{OH}\left(4_{2} \rightarrow 3_{1} E\right)$ line is offset by $49.4 \mathrm{~km} \mathrm{~s}^{-1}$. In the $146 \mathrm{GHz}$ spectra, the $\mathrm{HC}_{3} \mathrm{~N}(16 \rightarrow 15)$ line is offset by $86.46 \mathrm{~km} \mathrm{~s}^{-1}$ from the $\mathrm{H}_{2} \mathrm{CO}\left(2_{02} \rightarrow 1_{01}\right)$ emission. The curves show the Gaussian fit to each individual line as well as the spectrum resulting from a superposition of all identified lines: $\mathrm{a}=\mathrm{H}_{2} \mathrm{CO}\left(2_{02} \rightarrow 1_{01}\right), \mathrm{b}=\mathrm{HC}_{3} \mathrm{~N}(16 \rightarrow 15), \mathrm{c}=\mathrm{H}_{2} \mathrm{CO}\left(3_{21} \rightarrow 2_{20}\right), \mathrm{d}=$ $\mathrm{H}_{2} \mathrm{CO}\left(3_{22} \rightarrow 2_{21}\right), \mathrm{e}=\mathrm{CH}_{3} \mathrm{OH}\left(4_{2} \rightarrow 3_{1} E\right), \mathrm{f}=\mathrm{H}_{2} \mathrm{CO}\left(3_{03} \rightarrow 2_{02}\right)$.

\section{The Properties of the Molecular Gas in M82}

As the most prominent nearby galaxy in the northern hemisphere with a nuclear starburst, M 82 has been the target of many investigations, including numerous molecular line studies [e.g. 3,18]. Figure 1 shows an optical image of the highly inclined galaxy and its prominent galactic wind outlined in the $\mathrm{H} \alpha$ emission (red). The molecular gas is concentrated in a circumnuclear ring around the center of the starburst, which is seen nearly edge-on. The target of our observations were the two lobes of the ring, which are easily spatially separable with single-dish telescopes. Tuning the receivers of the IRAM $30-\mathrm{m}$ telescope to $145.60 \mathrm{GHz}$ and $218.48 \mathrm{GHz}$, we detected the $\mathrm{H}_{2} \mathrm{CO}$ transitions $2_{02} \rightarrow 1_{01}, 3_{03} \rightarrow 2_{02}, 3_{22} \rightarrow 2_{21}$, and $3_{21} \rightarrow 2_{20}$ at $145.60 \mathrm{GHz}, 218.22 \mathrm{GHz}, 218.48 \mathrm{GHz}$, and $218.76 \mathrm{GHz}$, respectively (Fig. 2). Note that the latter three lines were observed simultaneously in a $1 \mathrm{GHz}$ wide spectrum, which eliminated uncertainties due to pointing errors, calibration issues or different beamwidths in the temperature-tracing line ratio $\mathrm{H}_{2} \mathrm{CO}\left(3_{03} \rightarrow 202\right) / \mathrm{H}_{2} \mathrm{CO}\left(3_{21} \rightarrow 2_{20}\right)$. The $\mathrm{HC}_{3} \mathrm{~N}(16 \rightarrow 15)$ line is also present in both $145.60 \mathrm{GHz}$ spectra, but easily separated from the nearby $\mathrm{H}_{2} \mathrm{CO}$ line. Our observations also revealed $\mathrm{CH}_{3} \mathrm{OH}\left(4_{2} \rightarrow 3_{1} E\right.$ ) emission in the spectrum of the northeastern lobe. The presence of methanol in the molecular lobes of M 82 was independently 

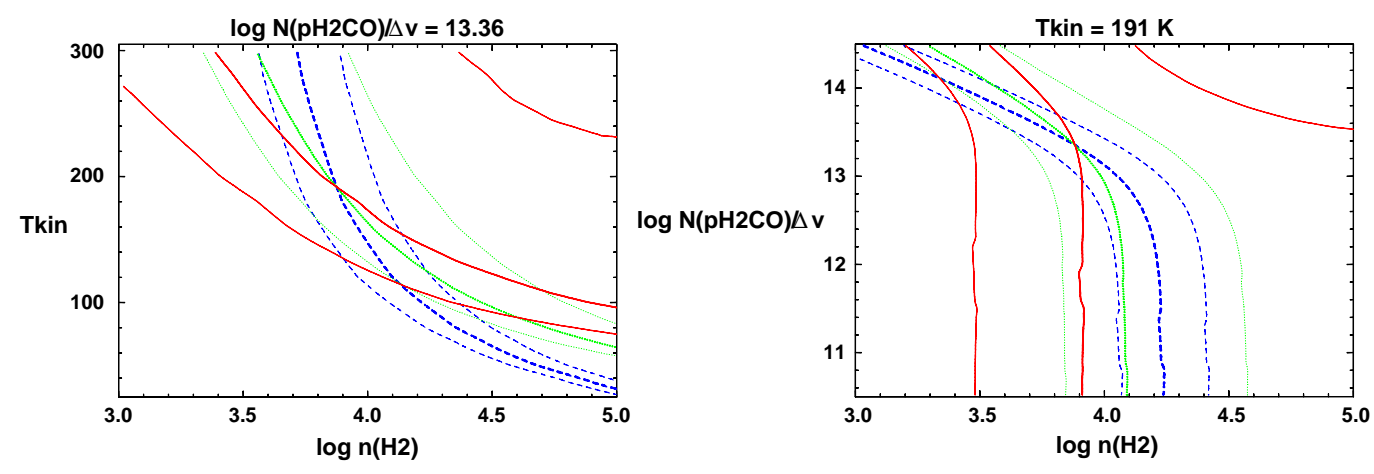

Figure 3: Results of our LVG analysis for the NE and SW lobes shown as cuts through the 3-D parameter space ( $T_{\text {kin }}$ in $\mathrm{K}, n_{\mathrm{H} 2}$ in $\mathrm{cm}^{-3}, N_{\mathrm{pH} 2 \mathrm{CO}} / \Delta v$ in $\mathrm{cm}^{-2} \mathrm{~km}^{-1}$ s) along a plane of constant para- $\mathrm{H}_{2} \mathrm{CO}$ column density per velocity interval (left) and of constant kinetic temperature (right). The lines represent the following line ratios: $\mathrm{H}_{2} \mathrm{CO}\left(3_{03} \rightarrow 202\right) / \mathrm{H}_{2} \mathrm{CO}\left(3_{21} \rightarrow 2_{20}\right)$ (red), $\mathrm{H}_{2} \mathrm{CO}\left(2_{02} \rightarrow 1_{01}\right) / \mathrm{H}_{2} \mathrm{CO}\left(3_{03} \rightarrow 2_{02}\right)$ (blue), $\mathrm{H}_{2} \mathrm{CO}\left(2_{02} \rightarrow 1_{01}\right) / \mathrm{H}_{2} \mathrm{CO}\left(3_{21} \rightarrow 2_{20}\right)$ (green). The corresponding dashed lines outline the uncertainties of each line ratio. The adopted source size is $\theta_{s}=7 . ! 5$ and the assumed para- $\mathrm{H}_{2} \mathrm{CO}$ abundance per velocity gradient is $\Lambda=1 \times 10^{-9} \mathrm{~km}^{-1}$ spc.

confirmed by [9].

The basic assumption that the observed emission lines all originate from the same volume of molecular gas with the same average physical properties implies that all lines should have the same velocity profile, parametrized here by a Gaussian curve with the same central velocity and the same line width for all lines. With these constraints, we fitted all detected lines of a spectrum simultaneously using the technique of "fitting dependent lines" in class, a part of the GILDAS software package. Accounting for the small difference in beam widths between the observations at $145.60 \mathrm{GHz}$ and those at $218.48 \mathrm{GHz}$, we derived the integrated line intensities of the detected $\mathrm{H}_{2} \mathrm{CO}$ lines and compared the line intensity ratios of our observations with the results of our nonLTE models. These models are based on the LVG approximation and a spherical cloud geometry and cover a wide parameter space in kinetic temperature, gas density and para- $\mathrm{H}_{2} \mathrm{CO}$ column density per velocity interval (see [11] for details). The comparison suggests that the average kinetic temperature of the molecular gas traced by the observed $\mathrm{H}_{2} \mathrm{CO}$ lines is about $200 \mathrm{~K}$ in both lobes, a moderate gas density of $7 \cdot 10^{3} \mathrm{~cm}^{-3}$ and a total molecular gas mass of about $1.5 \cdot 10^{8} M_{\odot}$ per lobe (Fig. 3). While the exact numbers depend slightly on the $\mathrm{H}_{2} \mathrm{CO}$ abundance and the exact extent of the emission region, the kinetic temperature of the molecular gas is in any case much higher than the dust temperature of $48 \mathrm{~K}$ [2].

At first, a kinetic gas temperature of about $200 \mathrm{~K}$ may seem odd considering that the dust temperature is significantly lower. However, recent comprehensive studies of multiple CO lines suggest the presence of at least two distinct molecular gas components in M 82, where the highly excited gas component may be the dominant one $[19,8]$. In these studies, the physical properties of the high-excitation molecular gas phase are remarkably similar to our results, which were derived completely independently. Evidence for a warm molecular gas component has also been found in the analysis of highly excited ammonia lines in nearby starburst galaxies [10] and in the investigation of IR rotational $\mathrm{H}_{2}$ lines in a sample of starburst and Seyfert galaxies [17]. Shocks and strong $\mathrm{UV}$ and/or X-ray irradiation and cosmic-ray heating are among the heating mechanisms that are 

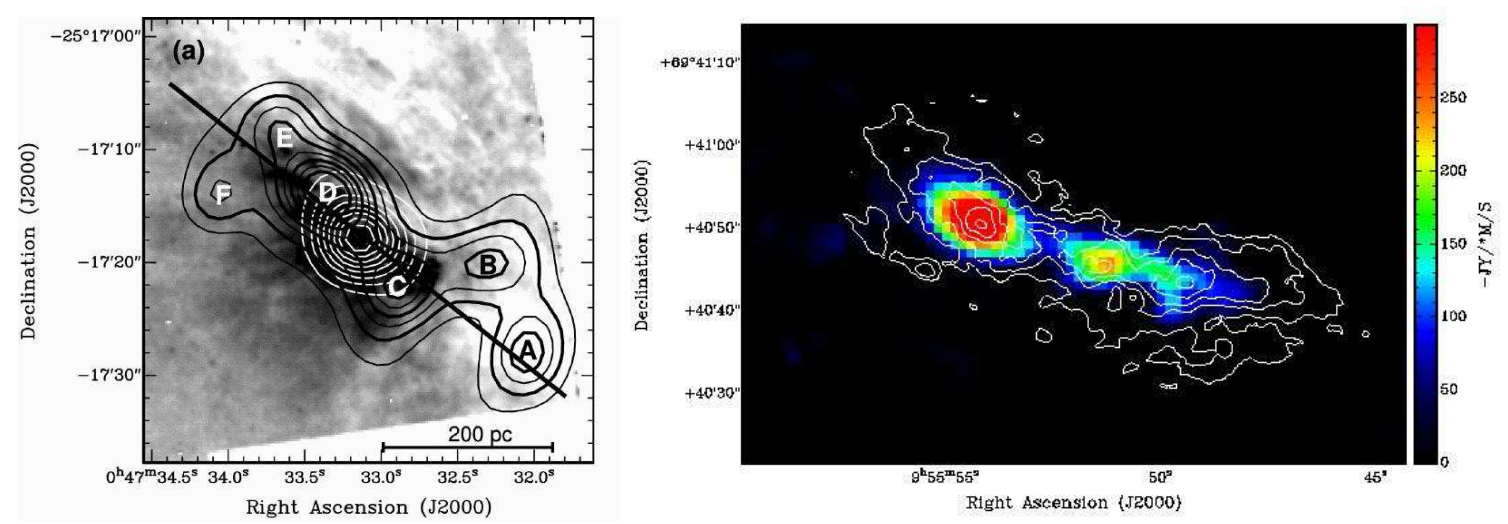

Figure 4: Left: Superresolved $\mathrm{NH}_{3}(3,3)$ emission displayed as black contours on a logarithmic HST WFPC2 F814W image of the core of NGC 253 [15]. Right: Preliminary map of the $\mathrm{H}_{2} \mathrm{CO}\left(1_{10} \rightarrow 1_{11}\right)$ line seen in absorption in the molecular ring of M82. The white contours outline the $\mathrm{CO}(2 \rightarrow 1)$ emission as mapped by [20].

likely to be found in active environments like starbursts and AGN and that can heat a large fraction of the molecular gas to temperatures of about $150 \mathrm{~K}[1,17]$.

\section{Conclusions and Outlook}

1. We have demostrated the diagnostic power of selected formaldehyde lines in deriving the physical properties of the molecular gas in external galaxies.

2. We detected several para- $\mathrm{H}_{2} \mathrm{CO}$ lines at $146 \mathrm{GHz}$ and at $218 \mathrm{GHz}$ in both molecular lobes of $\mathrm{M} 82$, including $\mathrm{H}_{2} \mathrm{CO}$ lines of the $K_{a}=2$ ladder.

3. Our non-LTE line ratio analysis suggests the presence of warm $(\sim 200 \mathrm{~K})$, moderately dense $\left(7 \cdot 10^{3} \mathrm{~cm}^{-3}\right)$ molecular gas near the nuclear starburst, which is in very good agreement with the properties of the high-excitation molecular gas component found in recent comprehensive studies of multiple CO lines.

4. Our case study supports the view that in active environments like starburst galaxies and AGN, a large fraction of the molecular gas may have a kinetic temperature of a few hundred Kelvin.

5. We find strong evidence for the presence of methanol in at least the northeastern lobe in M 82, confirming the results of [9].

Due to the instrumental limitations of the current telescopes and correlators, there are still very few investigations of the properties of the molecular gas in external galaxies at resolutions $<10^{\prime \prime}$ using sensitive temperature probes. NGC 253, a nearby starburst galaxy similar to M 82, has been observed with the ATCA in ammonia inversion lines (Fig. 4), which suggest kinetic temperatures of $140 \mathrm{~K}$ in the northeastern molecular cloud complexes and of $200 \mathrm{~K}$ in the southwestern complexes [15]. Preliminary results of high-resolution observations of $\mathrm{M} 82$ centered on the $\mathrm{H}_{2} \mathrm{CO}\left(1_{10} \rightarrow 1_{11}\right)$ line (Fig. 4) show the transition in absorption against the continuum [12]. The $\mathrm{H}_{2} \mathrm{CO}$ morphology 
and kinematics seem to follow the distribution and kinematics of the $\mathrm{CO}$ emission [20] with the exception of the southwestern lobe, which shows less $\mathrm{H}_{2} \mathrm{CO}$ absorption than the northeastern lobe.

With the commissioning of ALMA and the new correlator of the EVLA, we will be able to detect molecules like formaldehyde and ammonia with high spatial and frequency resolution in a large number of galaxies, employing their full diagnostic power to derive the physical properties of the molecular gas in galactic environments very different from the Milky Way disk and thus to reveal the interdependence of molecular gas, star formation and feedback processes.

\section{Acknowledgments}

We wish to thank the staff at Pico Veleta/Granada for their support during the observations. E.R.S. acknowledges a Discovery Grant from NSERC. This work has made use of the following software and resources: GILDAS, specx, Statistiklabor (FU Berlin, CeDiS), asyerr (Seaquist \& Yao), the JPL Catalog of spectral lines, The Cologne Database for Molecular Spectroscopy [12] and NASA's Astrophysics Data System Bibliographic Services (ADS).

\section{References}

[1] C.M. Bradford, T. Nikola, G.J. Stacey, A.D. Bolatto, J.M. Jackson, M.L. Savage, J.A. Davidson, \& S.J. Higdon, 2003, ApJ 586, 891

[2] J.W. Colbert, et al. 1999, ApJ 511, 721

[3] A. Fuente, S. García-Burillo, M. Gerin, J.R. Rizzo, A. Usero, D. Teyssier, E. Roueff, \& J. Le Bourlot, 2006, ApJ 641, L105

[4] Y. Gao \& P.M. Solomon, 2004, ApJ 606, 271

[5] D. Johnstone, A.M.S. Boonman, \& E.F. van Dishoeck, 2003, A\&A, 412, 157

[6] R.S. Klessen, M. Spaans, \& A.K. Jappsen, 2007, MNRAS 374, L29

[7] J.G. Mangum, \& A. Wootten, 1993, APJS 89, 123

[8] R.Q. Mao, C. Henkel, A. Schulz, M. Zielinsky, R. Mauersberger, H. Störzer, T.L. Wilson, \& P. Gensheimer, 2000, A\&A 358, 433

[9] S. Martín, J. Martín-Pintado, \& R. Mauersberger, 2006, A\&A 450, L13

[10] R. Mauersberger, C. Henkel, A. Weiß, A.B. Peck, \& Y. Hagiwara, 2003, A\&A 403, 561

[11] S. Mühle, E.R. Seaquist, \& C. Henkel, 2007, ApJ 671, 1579

[12] S. Mühle, E.R. Seaquist, \& C. Henkel, 2009, in prep.

[13] H.S.P. Müller, F. Schlöder, J. Stutzki, \& G. Winnewisser, 2005, J. Mol. Struct. 742, 215

[14] NASA, ESA, and The Hubble Heritage Team (STScI/AURA), http: / / hubblesite.org

[15] J. Ott, A. Weiß, C. Henkel, \& F. Walter, 2005, ApJ 629, 767

[16] T. Paumard, et al. 2006, ApJ 643, 1011

[17] D. Rigopoulou, D. Kunze, D. Lutz, R. Genzel, \& A.F.M. Moorwood, 2002, A\&A 389, 374

[18] E.R. Seaquist, S.W. Lee, \& G.H. Moriarty-Schieven, 2006, ApJ 638, 148

[19] J.S. Ward, J. Zmuidzinas, A.I. Harris, \& K.G. Isaak, 2003, ApJ 587, 171

[20] A. Weiß, N. Neininger, S. Hüttemeister, \& U. Klein, 2001, A\&A 365, 571 